\title{
Capacity Based Multicast Channel Assignment in Wireless Mesh Network
}

\author{
Anju Singh", Karan Singh, Sandeep Sharma \\ School of Information and Communication Technology, Gautam Buddha University, Greater Noida, India \\ Email: *11pit012@gbu.ac.in, karan@gbu.ac.in, sandeepsharma@gbu.ac.in
}

Received May 2013

\begin{abstract}
Wireless mesh networking (WMN) is an emerging technology that enables multihop wireless connectivity to areas where wiring or installing cables is difficult or expensive. Multicast is a form of communication that delivers information from a source to a group of destinations. In a single-channel WMN, all nodes share and communicate with each other via the same channel. In such a network, the throughput capacity of multicast degrades significantly as the network size increases. A critical factor that contributes to this rapid degradation is the co-channel interference in single-channel WMNs. The major advantage of WMN is that power is not the major issue as compare to other wireless network like MANET, Sensor etc. Hence Power can be optimally utilized in WMN to increase throughput and total network efficiency. In this paper, we propose a channel assignment algorithm for multicast based on high channel capacity with minimum interference. This scheme uses all overlapping and non overlapping channel for the channel assignment. By this scheme we provide better performance in terms of average packet delivery ratio, average throughput and average end to end delay with respect to multichannel multicast channel assignment schemes.
\end{abstract}

Keywords: WMN; Multicast; Multi Interface; Non Overlapping Channels; Partially Overlapping Channels; SNR

\section{Introduction}

Wireless mesh network is also known as community wireless networks. It is an emerging technology that supports many important applications such as Internet access provisioning in rural areas, ad hoc networking for emergency and disaster recovery, security surveillance, and information services in public transportation systems. Due to its promising technology it is becoming the major avenue for the next generation of wireless mobility [7]. The technology enables networking capability where wiring or installing cables are difficult or expensive. Wireless mesh network (WMN) is a new cost effective technology which constructs a resilient, locally networked access to communication infrastructure. This is due to its desirable characteristics multi-hop routing, auto configuration, bandwidth fairness, low cost, easy deployment, self healing and self organized.

In the recent years, the demand for multicast TV, video conference and online multicast based games are hugely increased. More online programs that could be live soccer match or live performance made the multicast communication more important research topic in WMNs [8]. Some commercial deployments are already working

\footnotetext{
"Corresponding author.
}

to provide low-cost connectivity to residents and local businesses. Multicast is a form of communication that delivers information from a source to a group of destinations simultaneously in an efficient manner. Important applications of multicast include distribution of financial data, billing records, software, and newspapers; audio/ video conferencing; distance education; IP television; and distributed interactive games. Research on multicast in WMNs has considered mostly networks with a single channel, i.e., all nodes in the network share and communicate with each other via one single channel [4]. The study shows that the throughput capacity of a singlechannel WMN degrades significantly as the network size increases.

Traditionally, WMN were equipped with node having a single radio. They were faced with number of limitations such as lower throughput and limited use of available wireless channels [5]. Major issue of wireless network is its deployment so WMN are preferred over other wireless network due to its random assignment node and capacity enhanced feature. But, using single channel creates interference like hidden terminal problem and exposed terminal problem. So, this problem can be improved by using multi channel in wireless mesh network.

Multiple channels with multi radio are one of the most 
effective approaches to achieve higher throughput. The network throughput in these multichannel systems can be increased multiplicatively at the cost of additional interface equipment. The tremendous popularity of wireless networking in recent years has led to the commoditization of wireless radios whose prices have fallen dramatically thanks to technology advances and mass production [6]. Therefore, the idea of multi-interface multi-channel wireless networking is very promising, allowing us to use two or more radios on the same device. The network has n channels, which may either overlap, such that a channel partially shares its frequency spectrum with the adjacent channels, or may be completely separated (non-overlapping or orthogonal). Orthogonal channels do not interfere with each other.

This paper focuses on the channel allocation scheme which efficiently utilizes multiple wireless interfaces to achieve better throughput thereby increasing the network capacity. We are using the capacity based channel assignment in which channel are assigned on the basis of the link which has high capacity and have some channel difference to already assigned channel. On average, a multichannel wireless network at least doubles the throughput, since each node is now in full-duplex mode, being able to transmit and receive simultaneously. Multi-interface networks, in return, require efficient channel assignment (CA) and routing algorithms that can take advantage of multiple channels and multiple interfaces.

\section{Related Work}

Guokai et al. [1] proposed the channel assignment scheme through Ascending and Heuristic approach. In which initially construct the multicast tree using level channel assignment (LCM) and multichannel multicast (MCM) approach then assign the channels to it. Mesh network initially needs to convert in a spanning structure and Tree structure is the least complex structure. So by LCA and MCM form the tree. Level Channel Assignment (LCA) is a method to build a multicast tree. Initially, the nodes obtain their level information [2]. The BFS is used to traverse the whole network. All the nodes are portioned into different levels according to the hop count distances between the source and the nodes. If node a (in level i) and b (in level $i+1$ ) are within each other "s" communication range, then "a" is called the parent of "b", and "b" is called the child of "a". Then build a multicast tree based on the node level information. Initially, the source and all the receivers are included in the tree.

Then, for each multireceiver $\mathrm{v}$, if one of its parents is a tree node then connect it with that parent, and stop. Otherwise randomly choose one of its parents, say $\mathrm{fv}$, as relay node on the tree, and connect $\mathrm{v}$ and $\mathrm{fv}$. Afterwards, we try to find out the relay node for fv recursively. The process repeats until the entire multireceiver is included in the multicast tree.

The tree nodes decide their channel assignment with the level information.

- The source node (level 0) only uses one interface, which is assigned channel 0 . This interface is responsible for sending packets to the tree nodes in level 1 .

- The internal tree node in level $i(i \geq 1)$ uses two interfaces: one is assigned channel $i-1$, which is used to receive packets from the upper level; the other is assigned channel 1, which is used to forward the packets to the tree nodes at level $i+1$.

- The leaf in the level $\mathrm{i}(\mathrm{i} \geq 1)$ uses two interfaces: one uses channel $\mathrm{i}-1$ to receive the packets from level $\mathrm{i}$ -1 , the other uses channel $i$ to forward the packets to the mesh clients within the communication range that desire to receive the packets.

For example in Figure 1, the node $s$ is the source and nodes f, g, e are the multireceiver. In Figure 1 \{s, f, g, and e are included in the multicast tree. Since nodes of g's parents are tree nodes, it randomly selects $d$ as a parent node and connects node $g$ with $d$. Then choose d's parent $b$ as a tree node and connect $d$ with $b$. Since b's parent $\mathrm{s}$ is a tree node connect b with s. Next, we start from multireceiver e. Connect e with its parent node $b$ and stop because $\mathrm{b}$ is already connected with tree node $\mathrm{s}$. Similarly the third multireceiver $\mathrm{f}$, connect $\mathrm{f}$ with c, c with a and then a with s. Thus the tree construction is completed by connecting all the receivers with the tree.

Multichannel Multicast (MCM) is another approach to construct multicast tree in which the throughput increases effectively [3]. Here the main aim is to minimize the number of relay nodes and hop count distance between source and destination. When all the Nodes are multireceiver, the multicast problem becomes the broadcast problem. Broadcast is a special case of multicast. The broadcast structure in the mesh network is built by the following steps:

- After the BFS traversal, all the nodes are divided into different levels.

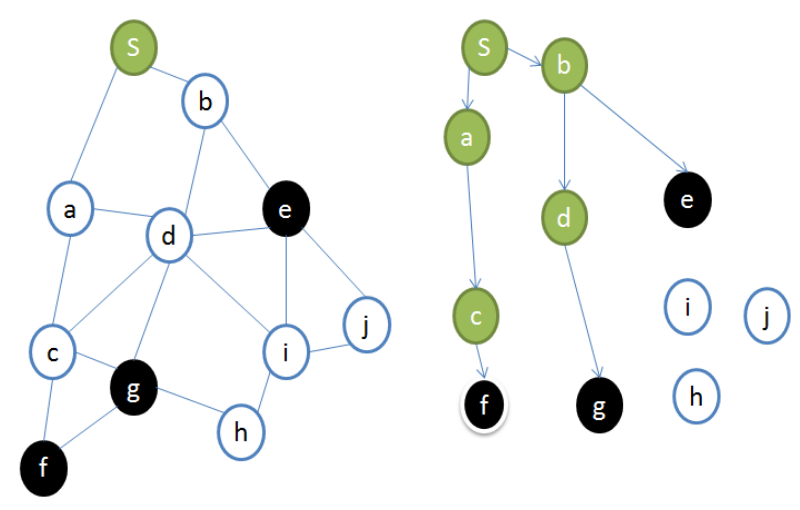

Figure 1. LCA mesh tree mesh. 
- Delete the edges between any two nodes of the same level, with which we get the elementary communication structure "tree mesh".

- Identify the minimal number of relay nodes that form the broadcast tree.

Using more relay node means more transmissions in the network. Because the number of available channel is limited, more transmissions would result in more interference and result in more bandwidth cost. Hence, minimizing the multicast tree size helps to improve the throughput. The purpose of this step is to identify the relay node for a node that has more than one parent nodes so that the number of relay node is minimal. In broadcast structure unnecessary branches are present if the destinations do not involve all the nodes. Hence, we propose to construct a structure using the MCM Tree Construction algorithm. The goal of the algorithm is to discover the minimal number of relay nodes needed to construct a multicast tree. The search process starts from the bottom to the top.

A simple example is shown to explain the process in a tree mesh in Figure 2, where nodes 6, 7, and 8 are the multireceiver. First select node 4 at level 2 because it covers all the multireceiver at level 3 . Next select node 2 at level 1, which covers all the multi receivers and the relay node at level 2. By doing these steps finally we get the multicast tree in Figure $\mathbf{2}$ because it covers all the multireceiver at level 3. Next select node 2 at level 1 , which covers all the multi receivers and the relay node at level 2. By doing these steps finally we get the multicast tree in Figure 2.

Ascending Approach: In ascending approach from top to down in the tree, the channels are assigned to the Interfaces in the ascending order until the maximum channel Number is reached, then start from channel 0 again. Each child node of a parent gets the same channel allocation. This approach avoids the situation that the same Channel is assigned to two nearby links that interfere with each other. The number above the node represents the channel number used for its RI, while the number below the node represents the channel number for its SI. In the algorithm, only the limited orthogonal Channels

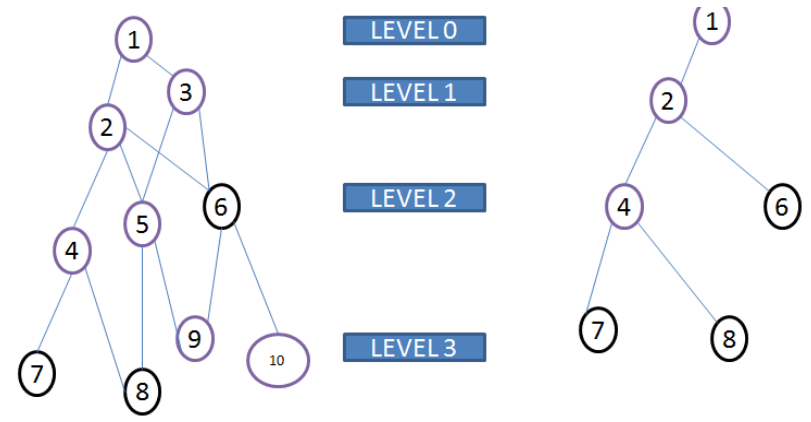

Figure 2. Multichannel multicast tree construction. are used. 802:11b provides 11 channels in American domain which are $5 \mathrm{MHz}$ apart in frequency. To be totally orthogonal, the frequency should be at least $30 \mathrm{MHz}$, so 802:11b can offer only three non-overlapping channels. Thus, although the Ascending Channel Allocation is easy to implement, its performance is still constrained by the limited number of orthogonal channels.

Heuristic Approach: In this, utilize all the channels available in band. Interference range decreases with channel separation. If physical distance is short b/w two wireless links then channel separation should be large. Here, main objective is to minimize the sum of interference area of all transmission. Bigger interference area means bigger chance two transmissions may interfere. When allocating a channel for relay node $u$, the channel assignment should take a channel that minimizes the sum of the square of the IRs between $\mathrm{u}$ and us neighbouring relay nodes, that is, minimize IR 2(uv) where N(u) represents the set of the neighbouring relay nodes of $u$. This is because the bigger interference area means the bigger chance two transmissions may interfere.

\section{Proposed Work}

The existing schemes of channel assignment in WMN used the non overlapping channels only and provide improvement in system performance characteristics like throughput, delay but wasted the limited resource. In the proposed work we will show the use of all available channels.

\subsection{System Model}

We model a WMN as a graph $\mathrm{G}(\mathrm{V}, \mathrm{E})$, with nodes $\mathrm{V}$ and links $\mathrm{E}$. Assume $\mathrm{T} \subseteq \mathrm{V}$ is the set of gateways. Each gateway has a high-bandwidth connection to the Internet, and can be viewed as a data source. Let $S$ be the set of data transmission sessions. We define five vectors of variables. The first four are: the vector of data flows $f$; the vector of multicast throughput $r$; the vector of link capacities c; and the power assignment vector $\mathrm{P}$. The last one is on channel assignment. We assume that each node is equipped with radio of capacity c. Here $\Gamma$ represents the set of pre-defined channels in the IEEE $802.11 \mathrm{~b} / \mathrm{g}$ standard.

There are many channel assignment schemes for WMN that are basically based on the channel separation concept. On the basis of channel separation, authors mainly defined that if channel separation between two adjacent channels is 4 or more than 4 then channel correlation coefficient is minimum. We know that interference factor between channels is directly proportional to channel correlation coefficient. So if channel correlation coefficient value is zero then interference factor will be zero. On the basis of this concept only some specific channels mostly 
near about 3 channels amongst 11 available channels of IEEE 802.11 standards are used. But, in our proposed scheme for each transmission we use one of the 11 channels of IEEE 802.11 standard. Now we proposed a channel scheme that is based on channel capacity and channel separation both. We know that channel correlation coefficient is inversely proportional to channel separation and channel interference is directly related to channel correlation coefficient. We analyse that if channel capacity is high then it can tolerate some amount of interference. We find that channel capacity not only depends upon the channel separation but also depends on some other factors. So, channel capacity of channel defined by the following equation

Where $\mathrm{b}$ bandwidth, Gee $=$ Gain, $\mathrm{Pe}=$ Power of channel $\mathrm{e}$, Ile $=$ Channel correlation coefficient, $\mathrm{Pl}=$ power of channel l, Gle = Interference coefficient between channel e and $1, \sigma 2=$ Noise associated with a link.

$$
\mathrm{C}=\operatorname{blog}_{2}\left(1+\mathrm{SINR}_{\mathrm{e}}\right)
$$

Where,

$$
\operatorname{SINR}_{\mathrm{e}}=\frac{\mathrm{G}_{\mathrm{ee}} \mathrm{P}_{\mathrm{e}}}{\sum_{1 \neq \mathrm{e}} \mathrm{I}_{1 \mathrm{e}} \mathrm{P}_{1} \mathrm{G}_{1 \mathrm{e}}+\sigma_{2}}
$$

So, we observe that my proposed work which is based on channel capacity show that when channel capacity is high, it can tolerate up to a threshold limit of channel interference factor.

\subsection{Procedure for Channel Assignment}

We are providing the procedure of proposed work in following way:

STEP 1 Create mesh network with the help of adjacency matrix. Set stack of all channels.

STEP 2 Given total no. of channels $C$ that is defined in IEEE 802.11 standards.

STEP 3 Now set the parameter for all channels, according to equation number 1 .

STEP 4 Use BFS algorithm for traversing node from source node to all destination nodes.

STEP 5 Assign any channel to source node $S$.

STEP 6 Now, calculate the current link capacity using Equation (1) for next all intermediate nodes between source to all destination and calculate the channel separation between assumed and already used channel.

STEP 7 Check the assignment requirement:

1) If calculated channel capacity is more than threshold capacity.

2) If channel separation is more than threshold.

STEP 8 Select channel $e$ for which Ce is maximum and channel separation is more.

STEP 9 Assign that channel Ce to current Node.

STEP 10 Repeat these steps 4 to 8 until all stacks are empty.

\section{Result Analysis}

\subsection{Simulation Environment}

The proposed work is simulated in QualNet in which a simulation environment is created for demonstrating the channel Assignment schemes for wireless mesh network.

\subsection{Performance Matrices}

Packet delivery, throughput and delay are major performance criteria for channel assignment schemes for wireless mesh network.

\subsection{Simulation Parameters}

We simulated a small network of 50 nodes uniformly distributed over a $1000 \mathrm{~m} \times 1000 \mathrm{~m}$ area shown in Figure 3. The transmission power and transmission range of each node were varying. We adapt the uniform distribution to distribute loads evenly and to minimize interference among routers. The number of nodes and the corresponding network size were chosen in such a way that there were no disjoint nodes or network partitions throughout the simulation. The uniform distribution of nodes, the network size was computed such that any one-hop neighboring nodes were within the transmission range of each other. We used the IEEE 802.11b standard at the physical layer with a transmission rate of $11 \mathrm{Mbits} / \mathrm{s}$. The IEEE 802.11 Carrier Sense Multiple Access with Collision Avoidance (CS-MA/CA) is chosen as the medium access control (MAC) for multicast transmissions.

Each multicast group has one source. The source of a multicast group transmits at a constant bit rate properly set for each experiment. The numbers of multicast destinations (the group size) are also specified for each scenario. The source and the destinations of a multicast group were selected randomly. All destinations joined a multicast group at the beginning and stayed until the whole group terminated. In each experiment, the source sent data for 300 seconds of simulated time, at a constant bit rate specified for each experiment. After the source finished sending, the simulation continued to run for 100 seconds of simulated time to give the last packets time to be processed and routed, for a total of 400 seconds. This 400 -second duration does not include the time needed for constructing the routing tree at the beginning.

\subsection{Experiment Scenario}

We measured the average packet delivery ratio, end to end delay and throughput as functions of Multicast Source Rate, Multicast Group Size, Number of Channels

The source rate was varied from 10 to 100 packet s/s. The total number of channels, including overlapping and non-overlapping channels, was 11 . 


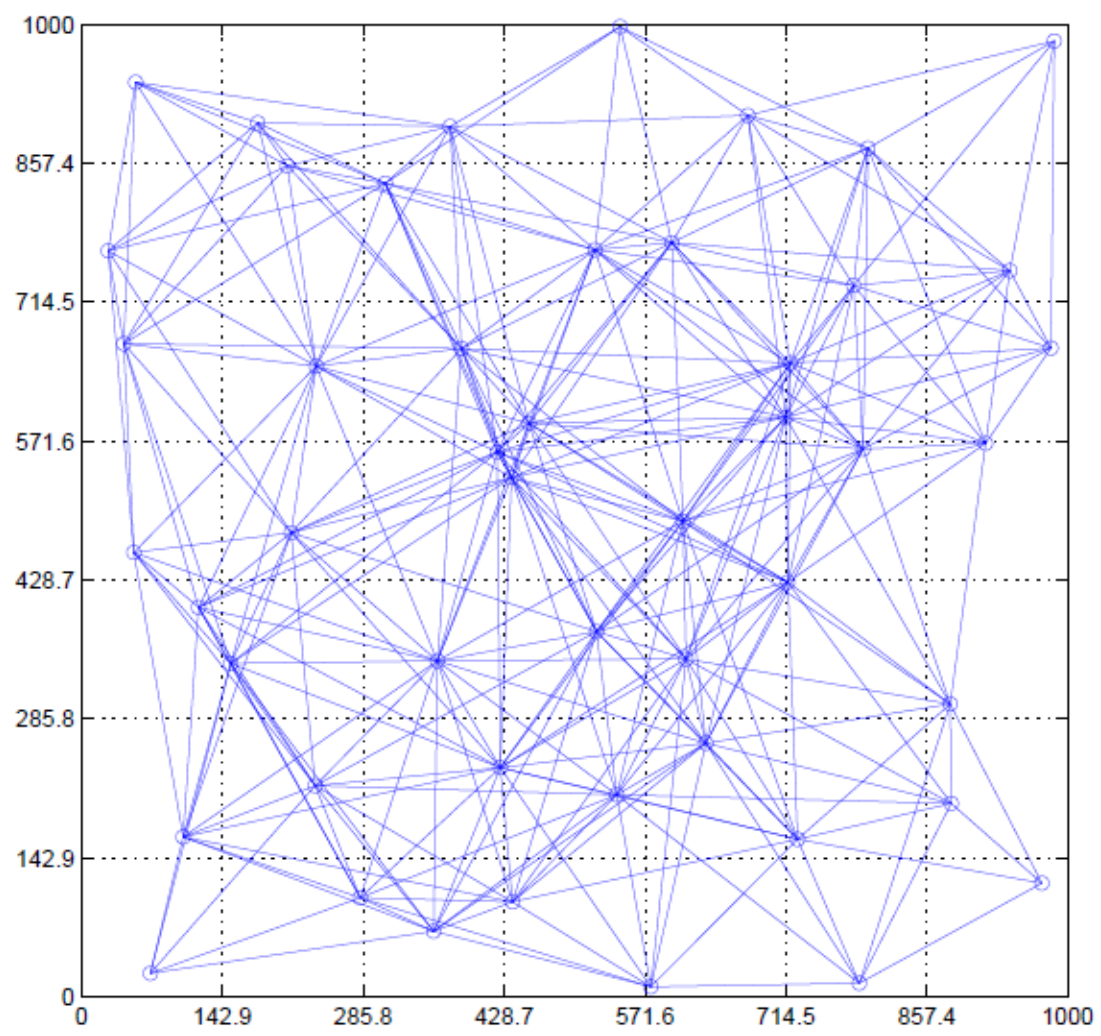

Figure 3. Network topology.

The number of multicast destinations in the multicast group ranged from 1 to 30 nodes in the 50-node network. The multicast source rates were set at 60 packets/s and 40 packets/s in the small and medium-size networks, respectively. Number of channels: The total number of overlapping and non-overlapping channels was varied from 1 to 20 . In the small network of 50 nodes, there were 20 multicast destinations, and the multicast source rate was set at 60 packet s/s.

\subsection{Function of Multicast Source Rate}

The sender's rate varies from 10 to 100 packets/sec. Multicast group is of 20 receivers in the network of 50 node. When traffic load is light (10 - 20 packets/sec) there is less contention and usage of channel, the multicast group did not take advantage of MCMR. A single channel is adequate for this case. When traffic load is moderate (above 40 packets/sec) the advantage of multichannel can be seen.

The Figure 4 shows that when the load of the traffic increases, packet delivery ration decreases. For both schemes MCM and CBM-CA we observe that as traffic load increases packet delivery ratio decreases. But we see that our CBM-CA based schemes give the better result as compare to MCM schemes because in our scheme, channel capacity is high at all link and also find that interference b/w channel also very low so the ratio of no.

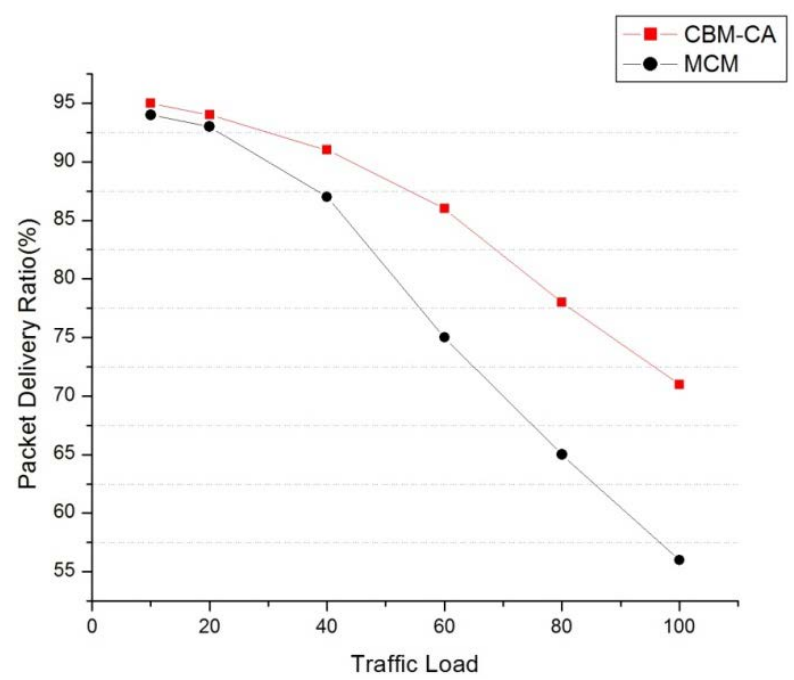

Figure 4. Average PDR.

of packet send and no. of packet receive is high means no. of packet deliver at each link. In the figure we see when traffic load is 10 packet/s then the packet delivery ratio for CBM-CA schemes near about $90 \%$ and when the traffic load is 100 packet/s the PDR is above $70 \%$ that is good in comparison to MCM.

The Figure 5 shows the average throughtput for the different traffic loads. We observe that that no. of packet received in given time interval increases as traffic load 


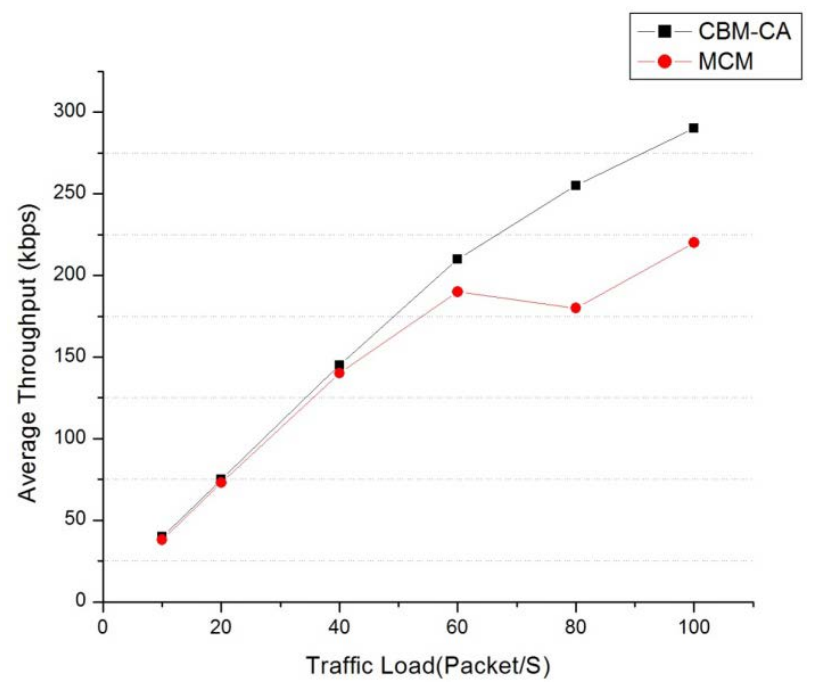

Figure 5. Average Throughput.

increases. In both schemes CBM-CA and MCM it happened that average throughput increases as traffic load increases. But in the case of CBM-CA provide better throughput as compare to MCM because no. of packet delivery rate of each channel in the multicast tree is high as compare to assign the channel in MCM schemes.

In the Figure 6 we observe that as the traffic load increases the average end to end delay some times increases and some time decreases. We observe that for the CBM-CA based schemes the end to end delay is near about $42 \mathrm{~ms}$ for the traffic load 10 to 100 packet/s and for MCM $44 \mathrm{~ms}$ so we find the end to end delay in case of CBM-CA gives better result due to high capacity. For all three cases, as the sender's rate increases, the throughput increases as expected; the PDR decreases because higher loads cause more congestion and collisions, resulting more packets dropped or damaged.

\subsection{Function of Number of Channels}

The number of channels in this set of experiments is varied from 1 to 20 . The multicast group in the 50-node network has 20 receivers, and its source sends at a rate of $60 \mathrm{pkts} / \mathrm{s}$. This rate yields a moderate load for the given group size in this network.

Figure 7 shows that as the no. of channel increases the packet delivery rate ratio will increase. In the figure we observe that the CBM-CA based schemes show the better result as compare to MCM based channel assignment scheme, as the no. of channel increases the no. of non overlapping channel increases and interference decreases so that PDR increases. In the case of CBM-CA capacity of each link also high with minimum interference so that gives the better result as compare to MCM.

The performance of CBM-CA is only slightly better than of MCM in this set of experiments. In the Figure 8

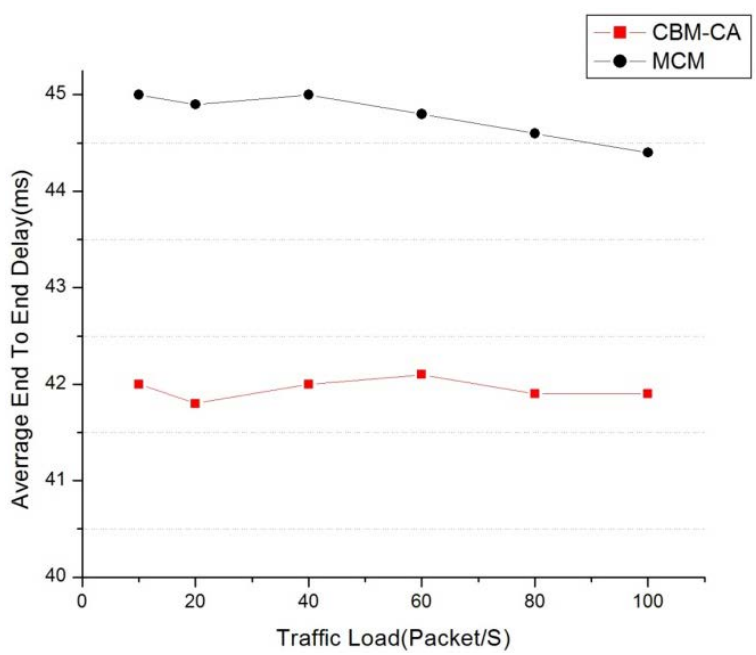

Figure 6. Average End to End Delay.

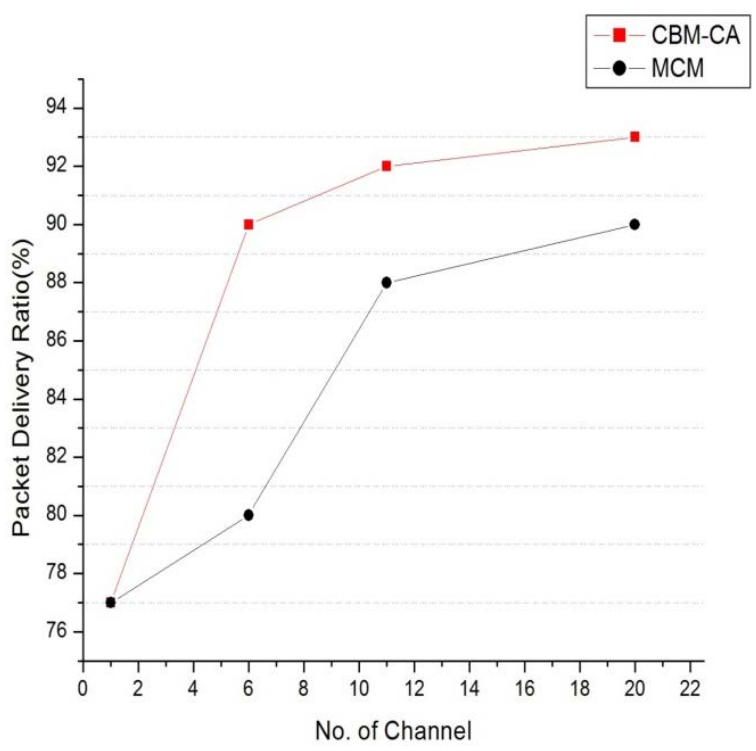

Figure 7. Packet delivery ratio.

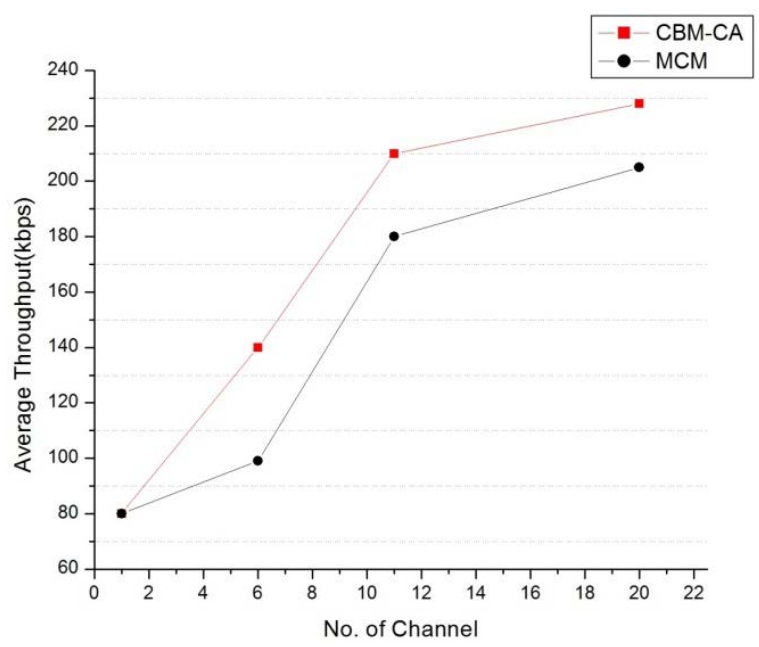

Figure 8. Average throughput. 
shows the average Throughput for different channels. We observe that average throughput increases as no. of channel increases and see that CBM-CA gives the better result as compare to MCM.

\section{Conclusion}

In this paper, we proposed the capacity based multicast channel assignment (CBM-CA) algorithm. The optimization function of the CBM-CA algorithm uses channel separation and channel capacity and thus does not rely on the computation of the interference factors. Advantages of our proposed algorithm include its simple implementation and high performance. The effectiveness of the CBM-CA algorithm is maximized in a network where the multicast group (tree) is dense. In such environment, the number of neighbouring nodes around a node is high and thus, without a carefully designed channel assignment (CA) algorithm like CBM-CA, the probability of channel conflicts among nodes would be very high. Our simulation results showed that the CBM-CA algorithm outperforms MCM in terms of average PDR, throughput, and end-to-end delay under various traffic loads, group sizes and different number of channels. We introduced an approach based on channel capacity to address the CA problem in multicast WMN to maximize throughput. Our simulation results showed that the CBM-CA algorithm outperforms MCM in terms of average PDR, throughput, and end-to-end delay under various traffic loads, group sizes and different number of channels.

\section{REFERENCES}

[1] G. K. Zeng, B. Wang, Y. Ding, L. Xiao and M. W. Mutka, "Efficient Multicast Algorithms for Multichannel Wireless Mesh Networks,” IEEE Transactions On Parallel And Distributed Systems, Vol. 21, No. 1, 2010. http://dx.doi.org/10.1109/TPDS.2009.46

[2] S. Sobana and S. Krishna Prabha, “An Efficient Method For Multichannel Wireless Mesh Networks With Pulse Coupled Neural Network," (IJACSA) International Journal of Advanced Computer Science and Applications, Vol. 3, No. 1, 2012.
[3] J.-W. Lin and S.-M. Lin, “Channel Assignment for Multicast in Wireless Mesh Networks with Considering Forwarding Weight and Distance”, 978-1-4244-8728-8/11/ $\$ 26.00$ (C)2011 IEEE.

[4] Z. Y. Yin, Z. C. Li and M. Chen, "A Novel Channel Assignment Algorithm for Multicast in Multi-radio Wireless Mesh Networks,”1-4244-1521-7/07/\$25.00 §2007 IEEE

[5] H. Skalli, S. Ghosh and S. K. Das, "Channel Assignment Strategies for Multiradio Wireless Mesh Networks: Issues and Solutions,” IEEE Communications Magazine, 2007. http://dx.doi.org/10.1109/MCOM.2007.4378326

[6] K. Ramachandran, E. M. Belding, K. Almeroth and M. Buddhiko, "Interference-Aware Channel Assignment in Multi-Radio Wireless Mesh Networks," Proceedings of IEEE INFOCOM, 2006. http://dx.doi.org/10.1109/INFOCOM.2006.177

[7] J. Tang, G. Xue and W. Zhang, "Maximum Throughput and Fair Bandwidth Allocation in Multi-Channel Wireless Mesh Networks," Proceedings of IEEE INFOCOM, 2006.

[8] I. Akyildiz, X. D. Wang and W. L. Wang, "Wireless mesh networks: A Survey,” Computer Networks, 2005. http://dx.doi.org/10.1016/j.comnet.2004.12.001

[9] K. Ramachandran, E. M. Belding, K. Almeroth and M. Buddhiko, "Interference-Aware Channel Assignment in Multi-Radio Wireless Mesh Networks," Proceedings of IEEE INFOCOM, 2006. http://dx.doi.org/10.1109/INFOCOM.2006.177

[10] J. Tang, G. Xue and W. Zhang, "Maximum Throughput and Fair Bandwidth Allocation in Multi-Channel Wireless Mesh Networks," Proceedings of IEEE INFOCOM, 2006.

[11] J. Tang, G. L. Xue and W. Y. Zhang,” InterferenceAware Topology Control and QoS Routing in MultiChannel Wireless Mesh Networks," Proceedings of the Sixth International Symposium on Mobile Ad Hoc Networking and Computing (MobiHoc 2005), Urbana-Champaign, 2005, pp. 68-77.

[12] R. Ramanathan, "Challenges: A Radically New Architecture for Next Generation Mobile Ad Hoc Networks," Proceedings of the Eleventh Annual International Conference on Mobile Computing and Networking (MobiCom 2005), Cologne, 2005, pp. 132-139. 